OPEN ACCESS

Edited by:

Heikki Olavi Tikkanen,

University of Helsinki, Finland

Reviewed by:

Antti M. Kiviniemi,

University of Oulu, Finland

Ilkka H. A. Heinonen,

University of Turku, Finland

${ }^{*}$ Correspondence:

Johannes J. Van Lieshout

j.j.vanlieshout@amc.uva.n

${ }^{\dagger}$ Björn J. P. Van Der Ster orcid.org/0000-0001-5190-5721

† Johannes J. Van Lieshout orcid.org/0000-0002-3646-2122

${ }^{\dagger}$ Berend E. Westerhof orcid.org/0000-0003-4753-2461

†Nicolaas H. Sperna Weiland orcid.org/0000-0003-1305-1846

Specialty section:

This article was submitted to Clinical and Translational Physiology, a section of the journal Frontiers in Physiology

Received: 04 October 2017 Accepted: 20 March 2018 Published: 09 April 2018

Citation:

van Der Ster BJP, Sperna Weiland NH, Westerhof BE, Stok WJ and van Lieshout JJ (2018) Modeling Arterial Pulse Pressure From Heart Rate During Sympathetic Activation by Progressive Central Hypovolemia.

Front. Physiol. 9:353 doi: 10.3389/fphys.2018.00353

\section{Modeling Arterial Pulse Pressure From Heart Rate During Sympathetic Activation by Progressive Central Hypovolemia}

\author{
Björn J. P. van Der Ster ${ }^{1,2,3+}$, Nicolaas H. Sperna Weiland ${ }^{3,4 t}$, Berend E. Westerhof ${ }^{3,5 t}$, \\ Wim J. Stok ${ }^{2,3}$ and Johannes J. van Lieshout ${ }^{1,2,3,6 * t}$
}

${ }^{1}$ Department of Internal Medicine, Academic Medical Center, Amsterdam, Netherlands, ${ }^{2}$ Department of Medical Biology, Academic Medical Center, Amsterdam, Netherlands, ${ }^{3}$ Laboratory for Clinical Cardiovascular Physiology, Academic Medical Center, Amsterdam, Netherlands, ${ }^{4}$ Department of Anesthesiology, Academic Medical Center, Amsterdam, Netherlands, ${ }^{5}$ Department of Pulmonary Diseases, Medical Center, Institute for Cardiovascular Research, VU University Amsterdam, Amsterdam, Netherlands, ${ }^{6}$ School of Life Sciences, The Medical School, MRC/Arthritis Research UK Centre for Musculoskeletal Ageing Research, Queen's Medical Centre, Nottingham, United Kingdom

Heart rate $(\mathrm{HR})$ has an impact on the central blood pressure $(\mathrm{BP})$ wave shape and is related to pulse wave velocity and therefore to timing and duration of systole and diastole. This study tested the hypothesis that in healthy subjects both in rest and during sympathetic stimulation the relation between $\mathrm{HR}$ and pulse pressure (PP) is described by a linear effect model. Forty-four healthy volunteers were subjected to sympathetic stimulation by continuous lower body negative pressure (LBNP) until the onset of pre-syncopal symptoms. Changes in PP and HR were tracked non-invasively and modeled by linear mixed effect (LME) models. The dataset was split into two groups: the first was used for creating a model and the second for its evaluation. Models were created on the data obtained during LBNP. Model performance was expressed as absolute median error (1st; 3rd quantiles) and bias with limits of agreement (LOA) between modeled and measured PP. From rest to sympathetic stimulation, mean BP was maintained while HR increased ( $30 \%)$ and PP decreased gradually ( 20\%). During baseline, PP could be modeled with an absolute error of $6(4 ; 10) \mathrm{mm} \mathrm{Hg}$ and geometric mean ratio of the bias was 0.97 (LOA: 0.8-1.1). During LBNP, absolute median model error was $5(4 ; 8) \mathrm{mmHg}$ with geometric mean ratio 1.02 (LOA: 0.8-1.3). In conclusion, both during rest and during sustained sympathetic outflow induced by progressive central hypovolemia, a LME model of HR provides for an estimate of PP in healthy young adults.

Keywords: heart rate, linear mixed effect models, lower body negative pressure, pulse pressure, sympathetic stimulation

\section{INTRODUCTION}

In the resting condition an elevated arterial pulse pressure (PP), the pulsatile component of blood pressure (BP), usually reflects decreased arterial compliance specifically in the elderly and is then associated with multiple adverse cardiovascular outcomes. In contrast, when healthy subjects exercise PP increases by the combined effects of an increase in systolic pressure concomitant to the elevated cardiac output $(\mathrm{CO})$ and a reduction in diastolic pressure related to the vasodilatation 


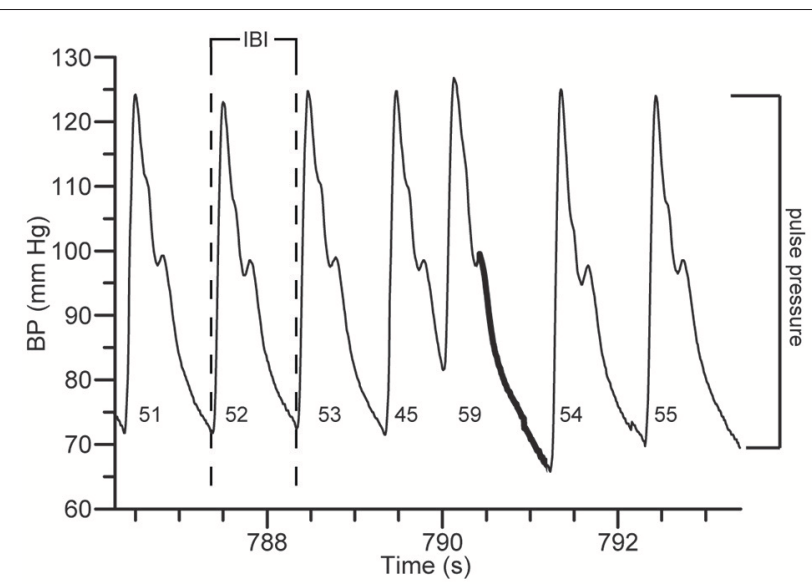

FIGURE 1 | Arterial pressure parameters. Pulse pressure (PP) is indicated for each beat at the root of each beat (in $\mathrm{mmHg}$ ). Note the effect of increased interbeat interval (IBI) (and thus decreased heart rate) on diastolic pressure runoff (indicated in bold) increasing PP. The preceding beat shows the reverse effect: due to a premature ventricular contraction (reducing the IBI, increasing heart rate), PP decreases.

in the exercising muscles (Lewis et al., 1983; Kim et al., 2015). PP provides prognostic utility beyond that of mean arterial pressure (MAP) (Selvaraj et al., 2016) and respiratory variations in PP relate to the magnitude of the central blood volume or left ventricular preload (Michard, 2005; Bronzwaer et al., 2015). However tracking PP requires a continuous BP signal either through arterial cannulation or through non-invasive BP measurement by plethysmography (Martina et al., 2012).

The duration of the cardiac cycle determines the length of diastole and influences the shape of the arterial pressure waveform (Wilkinson et al., 2000; Lantelme et al., 2002; Albaladejo et al., 2004; Haesler et al., 2004; Westerhof et al., 2008; Mackenzie et al., 2009; Benetos et al., 2010; Westerhof and Westerhof, 2013; Rimoldi et al., 2016). Recently, studies in both animals and humans using pacing or a selective negative chronotropic agent alleged evidence for an inverse relationship between PP and heart rate (HR) (Lantelme et al., 2002; Albaladejo et al., 2004; Haesler et al., 2004; Rimoldi et al., 2016). A lower HR prolongates diastole more than systole, widening PP (Figure 1; Folkow and Ely, 1998).

This study tested the hypothesis that in healthy subjects both in rest and during sympathetic stimulation the relationship between HR and PP can be described by a linear mixed effect (LME) model. To that purpose we tested this in young healthy adults by validating the resulting model on measured PP. Changes in HR and PP were introduced by sympathetic stimulation through induction of progressive central hypovolemia by lower body negative pressure (LBNP).

\section{MATERIALS AND METHODS}

This study was carried out in accordance with the recommendations of Academic Medical Centre Amsterdam medical ethical committee with written informed consent from all subjects. All subjects gave written informed consent in accordance with the Declaration of Helsinki. The protocol was approved by the Academic Medical Centre Amsterdam medical ethical committee (Study no. \#2014_310).

\section{Subjects}

Forty-four healthy, non-smoking volunteers (21 females) who performed regular exercise and that did not use cardiovascular medication participated in the study (age: 24, standard deviation (SD) 4 years; height: 177, SD $10 \mathrm{~cm}$; weight: 73, SD $11 \mathrm{~kg}$ ). Exclusion criteria were a medical history of cardio- and/or cerebrovascular disease, neurological disorders, diabetes mellitus, regular fainting and the use of medication. Prior to the experiment subjects abstained from heavy exercise, alcohol and caffeinated beverages for at least $12 \mathrm{~h}$.

\section{Instrumentation}

Continuous beat-to-beat BP was measured non-invasively using finger plethysmography (Nexfin, Edwards Lifesciences, Irvine, CA, USA; Martina et al., 2012). An appropriately sized finger cuff was applied to the mid-phalanx of the left hand. The hand was maintained at heart level. PP was defined as the difference between systolic and diastolic arterial pressure (SAP and DAP) and HR was defined as the inverse of the interbeat interval. MAP was the mean pressure over one BP pressure wave. Stroke volume (SV) was determined from the arterial pressure pulse wave by Nexfin CO-trek. Nexfin CO-trek readings are not different from a thermodilution estimate of $\mathrm{CO}$ for both invasive and noninvasive arterial pressure inputs, nor during upright exercise when compared to inert gas rebreathing (Bogert et al., 2010; Bartels et al., 2011) CO was SV multiplied by HR, and total peripheral resistance the ratio of MAP to CO.

\section{Protocol}

Following instrumentation, the lower part of the body was positioned inside a LBNP box (Dr. Kaiser Medizintechnik, Bad Hersfeld, Germany) and sealed at the level of the iliac crest (Goswami et al., 2009). The LBNP box was equipped with a saddle for subjects to sit on and to prevent leg muscle pump activation during the application of the sub-atmospheric pressure. Following $30 \mathrm{~min}$ of supine rest, continuous negative pressure (50 $\mathrm{mmHg}$ below atmospheric pressure) was applied to the lower body in one single step. The pressure inside the box was manually controlled and established within $20 \mathrm{~s}$.

\section{Monitoring of Progressive Central Hypovolemia}

Subjects were instructed to minimize movement and maintain their spontaneous breathing frequency and depth. Breathing frequency and end-tidal $\mathrm{CO}_{2}$ partial pressure were continuously monitored, verifying that end-tidal $\mathrm{CO}_{2}$ did not change significantly during the trial. An investigator experienced in human studies who was unoccupied by other experimental obligations monitored the subjects' well-being. LBNP was terminated either on request, at onset of (pre-) syncopal symptoms or after $30 \mathrm{~min}$. Pre-syncopal symptoms were defined 


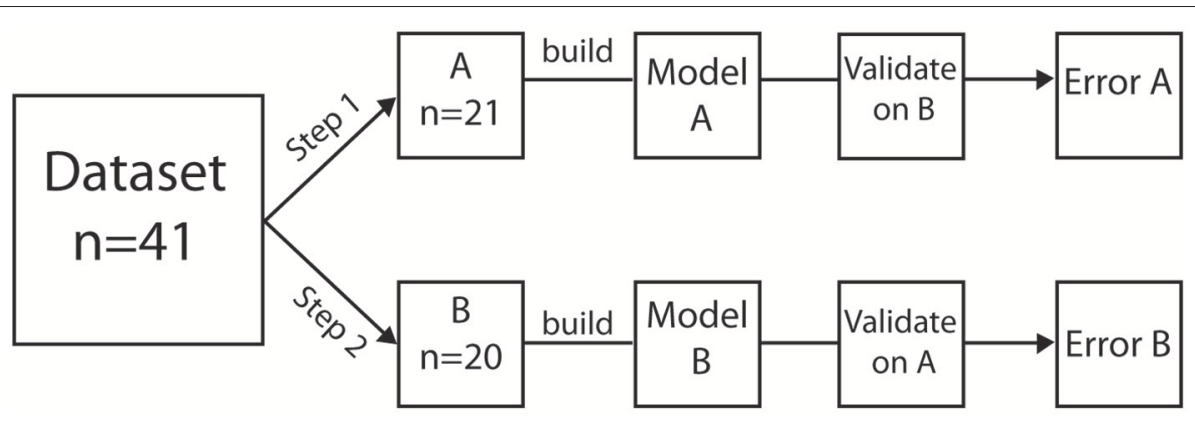

FIGURE 2 | Steps in analysis. Visual representation of the steps required to obtain model performance.

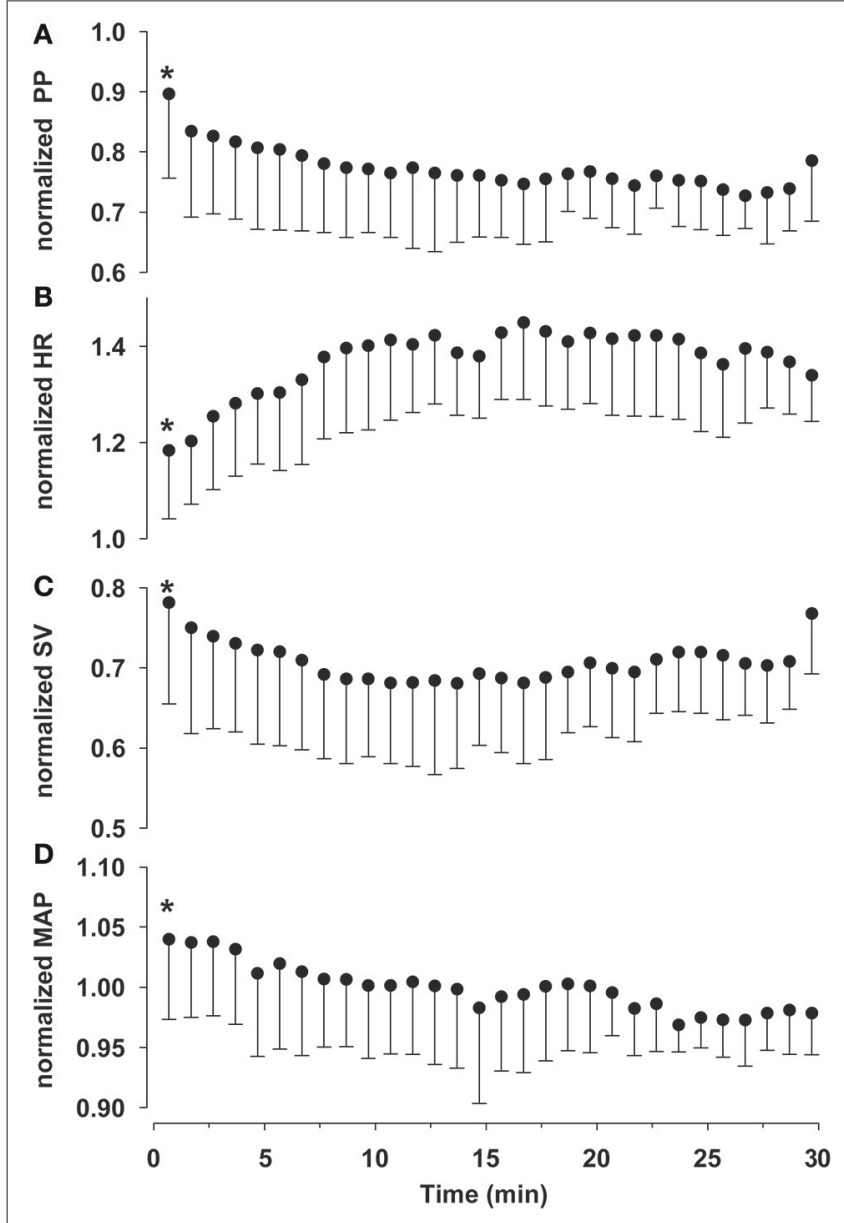

FIGURE 3 | Cardiovascular response to sympathetic stimulation by lower body negative pressure (LBNP). With subjects becoming pre-syncopal the number of subjects within this figure decreases over time. Values normalized to the values in baseline rest preceding LBNP. First minute was tested (paired $t$-test) for statistical significance and noted with an asterisk when different from baseline $(P<0.05)$. (A) PP, pulse pressure; (B) HR, heart rate; (C) SV, stroke volume (D) MAP, mean arterial pressure; Mean and standard deviation for each minute during LBNP.

as one or more of the following criteria: systolic arterial pressure below $80 \mathrm{mmHg}$, or a rapid drop in BP ( $\Delta$ systolic pressure $\geq 25 \mathrm{mmHg} / \mathrm{min}, \Delta$ diastolic arterial pressure $\geq 15 \mathrm{mmHg} / \mathrm{min}$ ), a drop in $\mathrm{HR}$ of $\geq 15 \mathrm{bpm}$, and/or sweating, light-headedness, nausea, vision alterations, skin pallor or a heat sensation.

\section{Analysis}

Data collected during the final $10 \mathrm{~min}$ of supine rest preceding onset of LBNP was averaged and defined as baseline. Data for each minute during LBNP was averaged and served as model input and output. We constructed LME models describing PP in relation to explanatory variable HR (fixed effect). Subject specific variables (random effects) were included to correct for, subject dependency and the expected variation in HR slope. LME models were computed in $\mathrm{R}$ (The $\mathrm{R}$ Project, The $\mathrm{R}$ Foundation for Statistical Computing, Vienna, Austria) (R Core Team, 2014) using the NLME library (Pinheiro et al., 2014). We used a crossover design to test model performance. The dataset was split into two groups (A and B). Initially, the data of group A served as model input and the data of group B was used to assess the performance of the acquired model. Then the process was interchanged: the data of group B was used to build a model and data of group A to test it (Figure 2). Absolute model errors determined during both baseline and LBNP were assessed per heartbeat. Median errors from all subjects following completion of the interchanging datasets are reported. Except for the modeling all data processing and statistics were performed in Matlab (Matlab 2007b, The MathWorks Inc., Natick, MA, USA).

\section{Calibration}

For the estimation of systolic and diastolic pressure the model was calibrated every $5 \mathrm{~min}$ to the 10 beat average of the noninvasive $\mathrm{BP}$ monitor to emulate an emergency setting in our hospital first aid department where on a routine basis only intermittent oscillometric BP is available.

\section{Statistics}

A rule of thumb for regression models requires 10 data samples for each included model parameter. Model performance is expressed as absolute median error, bias (mean of differences) and limit of agreement (LOA) (bias $\pm 1.96 \mathrm{SD}$ ) between measured and actual PP. In the case of normally distributed errors, bias and LOA are reported as geometric mean of the ratio between the two parameters. In the case of non-normally distributed model errors a 

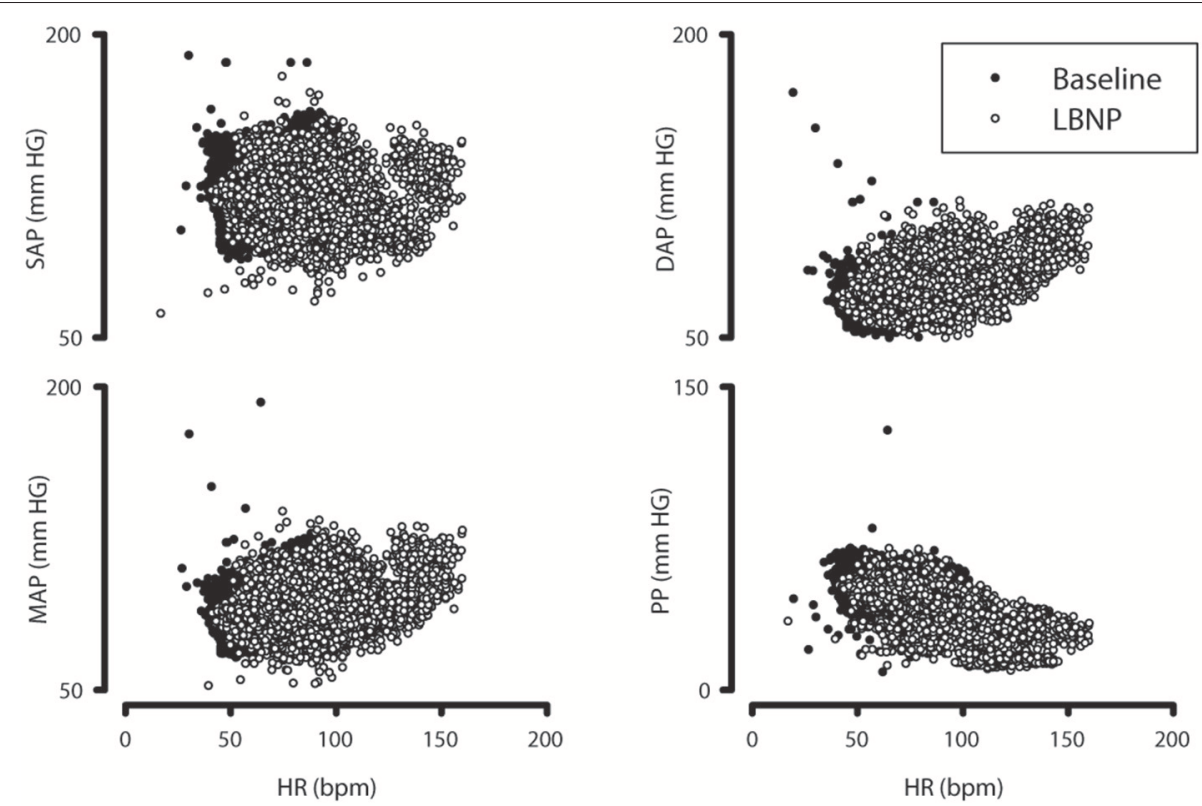

FIGURE 4 | Relationship between heart rate and systolic, diastolic, mean and pulse pressure during LBNP. Scatter plots of the raw data of systolic, diastolic, and mean blood pressure, and pulse pressure vs. heart rate (HR). There is a more distinct decrease of pulse pressure (PP, bottom right) between baseline (filled circles) and during lower body negative pressure (LBNP, open circles) compared to systolic and mean arterial pressure (SAP and MAP).

logarithmic transformation was performed on the data before computing bias and LOA followed by a transformation back to get the geometric means ratio and its LOA (Bland and Altman, 1999). Data is reported as means and SD in the case of normally distributed parameters, and as median [1st; 3rd quantile] for parameters that were not distributed normally.

\section{RESULTS}

Data of 3 subjects was excluded because of artifacts or noise in the measured signals, leaving data from 41 subjects available for analysis.

LBNP induced a rapid decline in SV $(\sim 25 \%)$ and PP $(\sim 15 \%)$ accompanied by an immediate $\sim 30 \%$ increase in $\mathrm{HR}$ (Figure 3). Mean BP was maintained whereas systolic pressure declined $\sim 10 \%$ and total peripheral resistance increased $\sim 20 \%$. LBNP was terminated in 37 out of 44 subjects because of pre-syncopal symptoms. Mean time until pre-syncope was 837 (SD 368) s; 7 subjects completed the full $30 \mathrm{~min}$ of LBNP. The relationships of $\mathrm{HR}$ and the $\mathrm{BP}$ parameters during baseline and LBNP (SAP, DAP, MAP and PP) are shown in Figure 4.

\section{Model Validation}

The interchanging of datasets resulted in two equations for the model:

$$
\begin{aligned}
& P P_{A}=67.30-0.35 \cdot H R \\
& P P_{B}=70.04-0.37 \cdot H R
\end{aligned}
$$

with $\mathrm{HR}$ in beats per minute and $\mathrm{PP}$ in $\mathrm{mmHg}$. The distribution of the model input for HR ranged from 58 (SD 9) to 98 (SD 15) bpm. Model within group residuals were 9.0; and 10.3 for group $A$ and $B$ respectively.

Absolute model errors were $6.0[3.9 ; 9.8]$ and $4.9[3.8 ; 7.6]$ $\mathrm{mmHg}$ (Figure 5) during baseline and LBNP respectively. Model errors were not normally distributed. Geometric mean ratio of the bias was 0.97 with LOA $0.81-1.13$ and 1.02 with LOA $0.77-1.27$ during baseline and LBNP respectively.

Calibration of the model to intermittent BP further reduced estimated PP errors to: 2 [2-4] and 3 [2-4] $\mathrm{mmHg}$ during rest and LBNP respectively. Errors of systolic and diastolic pressures were normally distributed (Table 1).

A concordance figure is shown in Figure 6. Mean degree to the line of identity was -5.5 CI $95 \%$ [ -0.13 to -10.8$]$ degrees. All but one sample fell within the range between -45 and -45 degrees. The sector holding $95 \%$ of the samples is \pm 30 degrees.

\section{DISCUSSION}

The novel finding of this study is that in a setting of a sustained increase in sympathetic outflow by LBNP PP can be estimated from a LME model based on HR. The implication is that in healthy subjects PP can be tracked over time from $\mathrm{HR}$ without the requirement of recording continuous (non-)invasive BP. The data indicates that such a model provides an acceptable estimate of PP when venous return becomes reduced with decreasing cardiac volume and filling pressures and enhanced peripheral vascular resistance. 


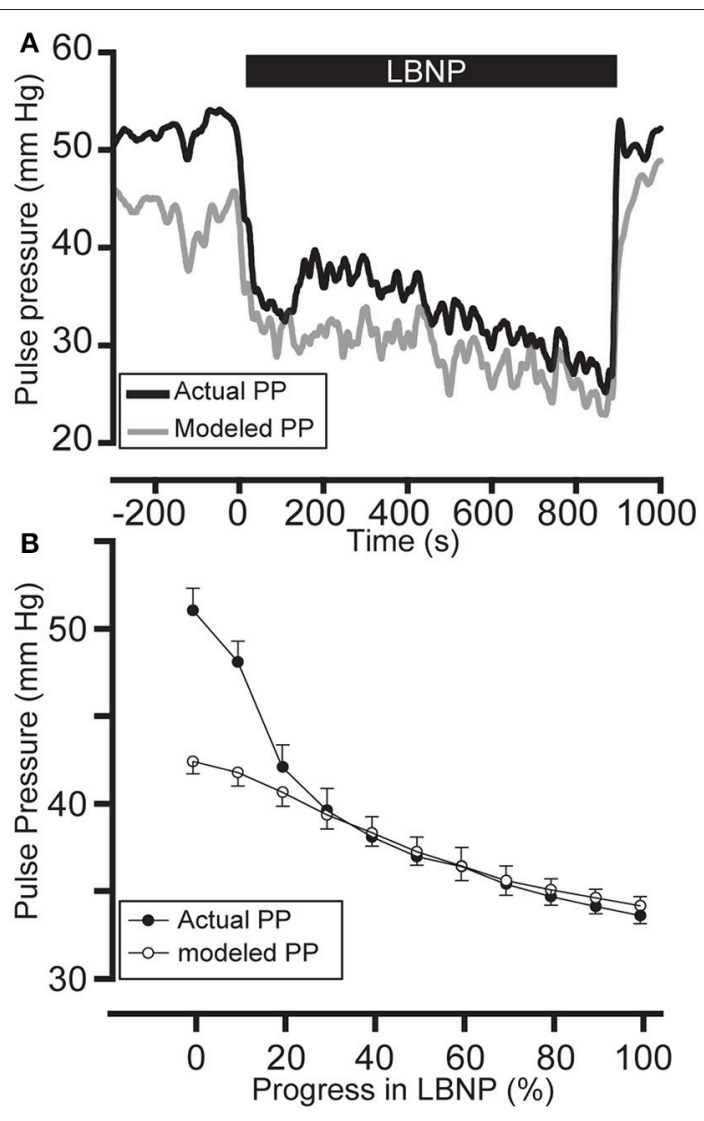

FIGURE 5 | Model performance. Pulse pressure estimated with linear mixed effect model of heart rate in one subject (A) and the entire study population (B). Measured (filled circles) and estimated (open circles) pulse pressure (PP) during lower body negative pressure (LBNP). Horizontal axis shows the progress of LBNP in seconds (A) and normalized to total duration of LBNP (B), i.e., till pre-syncope or LBNP lasting $30 \mathrm{~min}$. Note that the model misses initial non normally distributed part of the group mean PP due to the onset of LBNP with rapid decrease in PP in the first minute (as noted in Figure 3A). Mean and standard errors in 41 subjects.

The LBNP procedure was terminated as soon as a subject experienced pre-syncopal symptoms, and the LME models are thus based on a reducing number of samples per passing minute. A merit of LME models is that they allow for such repeated observations and are applicable to datasets with missing data and/or small sample size (Verbeke and Molenberghs, 2000; Gueorguieva and Krystal, 2004; Torng et al., 2007; Pan et al., 2012). Related to the linearity of the equation, our model can only describe the response as described here and not when the relationship between PP and HR reverses, which may occur during pre-syncope where regularly PP and HR decline rapidly. Since PP is inversely modeled to $\mathrm{HR}$ (Equation 1), a reduction in HR will incorrectly predict an increase of PP. A restriction of linear models is that they cannot describe such complexity and therefore become unreliable under conditions where the $\mathrm{PP}-\mathrm{HR}$ relationship is not in agreement with the model's assumptions, i.e., is an inverse linear one.
TABLE 1 | Mean bias, limit of agreement of blood pressure estimation during baseline and lower body negative pressure (LBNP).

\begin{tabular}{|c|c|c|c|c|}
\hline & \multicolumn{2}{|c|}{ Baseline } & \multicolumn{2}{|c|}{ LBNP } \\
\hline & Bias & $\begin{array}{c}\text { Limit of } \\
\text { agreement }\end{array}$ & Bias & $\begin{array}{c}\text { Limit of } \\
\text { agreement }\end{array}$ \\
\hline Systole & 0.71 [0.3-1.2] & 12.39 [11.5-13.2] & 1.66 [1.1-2.2] & 15.79 [14.8-16.8] \\
\hline Diastole & $0.34[0.1-0.6]$ & 9.03 [8.4-9.7] & $1.47[1.1-13.9]$ & $14.76[14.0-15.5]$ \\
\hline
\end{tabular}

Numbers reflect mean and 95\% confidence intervals in $\mathrm{mmHg}$.

\section{Limitations}

By design, all subjects were young, healthy individuals, which restrains us from extrapolating the data to aging patients with cardiovascular disease. The model was tested for a limited range of HRs during rest and LBNP and it is unlikely that the model maintains reported performance far beyond the reaches of the used range. The modeled PP (Figure 5B) has difficulty in predicting PP at the start of the LBNP protocol. Due to the almost stepwise fashion in which PP and SV reduce in response to LBNP, the model inherently cannot describe PP accurately at this early stage. Calculating a mean value during such a rapid change is invalid due to the non-normally nature of PP during this phase. This increases estimation error for the initial segment. In a real-life clinical setting, the occurrence of such a rapid drop in blood volume is unlikely.

\section{Clinical Perspective}

First line monitoring of patients presenting with hemorrhage includes measuring $\mathrm{HR}$, arterial oxygen saturation and noninvasive intermittent oscillometric blood pressure (NIBP). However, none of these represent biomarkers highly sensitive to the detection of a blood volume deficit since during the initial phase of hypovolemic shock mean BP is maintained by a baroreflex mediated increment in $\mathrm{HR}$ and total peripheral resistance (Grant and Reeve, 1941; Barcroft et al., 1944; McMichael, 1944; Ryan et al., 2012; Secher and Van Lieshout, 2016). In contrast, PP decreases progressively during hypovolemic shock and rather than mean BP reflects central blood volume due to its relation to SV (Convertino et al., 2006; Bighamian and Hahn, 2014). Since PP is an important biomarker for changing volume state, application of the reported model could assist in the monitoring of patients at risk for blood loss or development of hypovolemia. It is the rate of change (or trend) noticeable in $\mathrm{HR}$ and PP rather than their absolute values that contain the dynamic information essential for clinicians to decide whether the cardiovascular system is in steady-state or is still subject to change. PP is more closely related to flow parameters such as $\mathrm{SV}$ where a reduction in PP strongly suggests a decline in $\mathrm{SV}$. In general, BP is not sensitive for a reduction in flow, as illustrated in Figure 3: PP, SV and HR all change during LBNP, whereas MAP is still between 95 and 100\% of its initial value. 

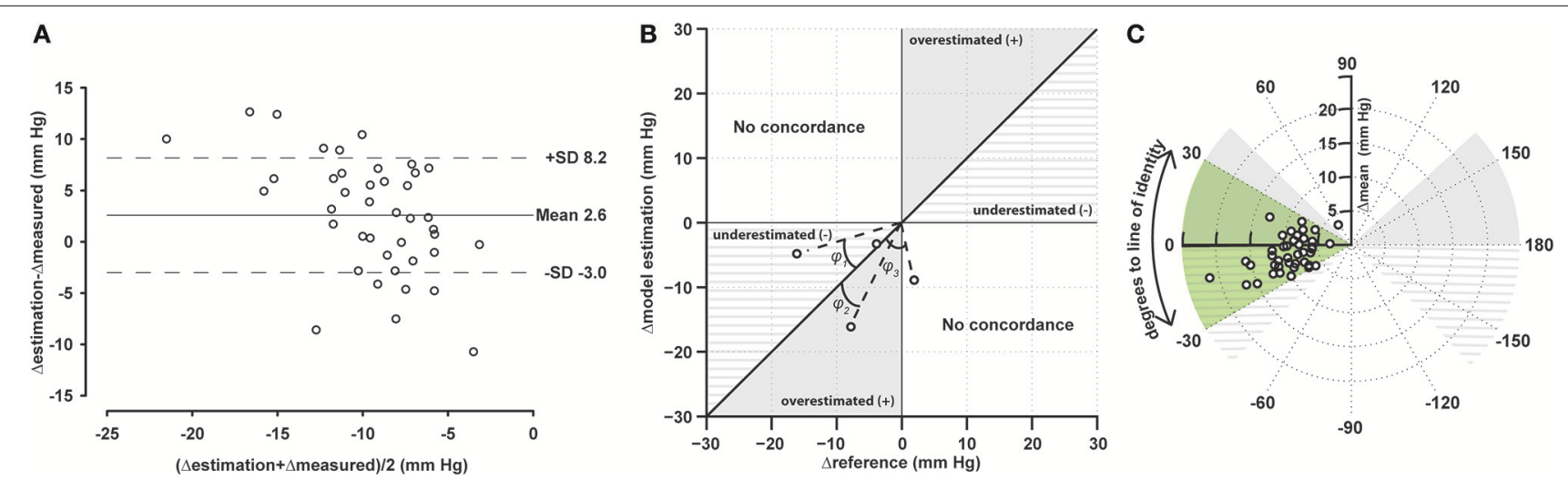

FIGURE 6 | Visualization of the difference in trend assessment during LBNP for both measures of pulse pressure. Bland-Altman (A) and polar plot

$(\mathbf{B}, \mathbf{C})$ representation of the difference between the measured and estimated pulse pressure trends. The visualized delta was computed using the mean during LBNP subtracted from the mean during baseline. The angle $\varphi$ toward the line of identity is either positive (overestimation) or negative (underestimation). $\varphi$ and the mean of both methods is translated to the polar plot (Critchley et al., 2011) (C). The shaded areas in panels (B,C) indicate overestimation of pulse pressure, whereas the hashed areas indicate underestimation of the pulse pressure. The green shaded area indicates the sector including $95 \%$ of the samples.

The presented model does not require additional devices or measurements to function and can be used whenever $\mathrm{HR}$ is available, e.g., from electrocardiography or pulse oximetry. Further we demonstrated that the model can upgrade intermittent oscillometric BP to a continuous tracking device of systolic and diastolic $\mathrm{BP}$ by a $5 \mathrm{~min}$ interval calibration.

In conclusion, in healthy young adults who progress from a resting situation to a situation of sympathetic activation by progressive central hypovolemia $\mathrm{PP}$ and its trend can be estimated from HR.

\section{REFERENCES}

Albaladejo, P., Challande, P., Kakou, A., Benetos, A., Labat, C., Louis, H., et al. (2004). Selective reduction of heart rate by ivabradine: effect on the visco-elastic arterial properties in rats. J. Hypertens. 22, 1739-1745. doi: 10.1097/00004872-200409000-00018

Barcroft, H., Edholm, O. G., McMichael, J., and Sharpey-Schafer, E. P. (1944). Posthaemorrhagic fainting. Study by cardiac output and forearm flow. Lancet 1, 489-491.

Bartels, S. A., Stok, W. J., Bezemer, R., Boksem, R. J., van Goudoever, J., Cherpanath, T. G., et al. (2011). Noninvasive cardiac output monitoring during exercise testing: nexfin pulse contour analysis compared to an inert gas rebreathing method and respired gas analysis. J. Clin. Monit. Comput. 25, 315-321. doi: 10.1007/s10877-011-9310-4

Benetos, A., Thomas, F., Joly, L., Blacher, J., Pannier, B., Labat, C., et al. (2010). Pulse pressure amplification a mechanical biomarker of cardiovascular risk. J. Am. Coll. Cardiol. 55, 1032-1037. doi: 10.1016/j.jacc.2009.09.061

Bighamian, R., and Hahn, J. O. (2014). Relationship between stroke volume and pulse pressure during blood volume perturbation: a mathematical analysis. Biomed. Res. Int. 2014:459269. doi: 10.1155/2014/459269

Bland, J. M., and Altman, D. G. (1999). Measuring agreement in method comparison studies. Stat. Methods Med. Res. 8, 135-160. doi: 10.1177/096228029900800204

Bogert, L. W., Wesseling, K. H., Schraa, O., Van Lieshout, E. J., de Mol, B. A., van Goudoever, J., et al. (2010). Pulse contour cardiac output derived from non-invasive arterial pressure in cardiovascular disease. Anaesthesia 65, 1119-1125. doi: 10.1111/j.1365-2044.2010.06511.x

\section{AUTHOR CONTRIBUTIONS}

Conception and experimental design: BS, BW, NS-W, JL, and WS; Data acquisition: BS and WS; Analysis: BS; Figure preparation: BS and JL Manuscript editing: BW, NS-W, WS, and JL.

\section{FUNDING}

Supported by an educational grant number Edwards Lifesciences: 2010B0797.

Bronzwaer, A. S., Ouweneel, D. M., Stok, W. J., Westerhof, B. E., and van Lieshout, J. J. (2015). Arterial pressure variation as a biomarker of preload dependency in spontaneously breathing subjects - a proof of principle. PLOS ONE 10:e0137364. doi: 10.1371/journal.pone.0137364

Convertino, V. A., Cooke, W. H., and Holcomb, J. B. (2006). Arterial pulse pressure and its association with reduced stroke volume during progressive central hypovolemia. J. Trauma 61, 629-634. doi: 10.1097/01.ta.0000196663. 34175.33

Critchley, L. A., Yang, X. X., and Lee, A. (2011). Assessment of trending ability of cardiac output monitors by polar plot methodology. J. Cardiothorac. Vasc. Anesth. 25, 536-546. doi: 10.1053/j.jvca.2011.01.003

Folkow, B., and Ely, D. (1998). Importance of the blood pressure-heart rate relationship. Blood Press 7, 133-138. doi: 10.1080/080370598 437321

Goswami, N., Grasser, E., Roessler, A., Schneditz, D., and Hinghofer-Szalkay, H. (2009). The cardiovascular response to lower body negative pressure in humans depends on seal location. Physiol. Res. 58, 311-318.

Grant, R. T., and Reeve, E. B. (1941). Clinical observations on air-raid casualties. Br. Med. J. 2, 293-297.

Gueorguieva, R., and Krystal, J. H. (2004). Move over anova: progress in analyzing repeated-measures data andits reflection in papers published in the archives of general psychiatry. Arch. Gen. Psychiatry 61, 310-317. doi: 10.1001/archpsyc.61.3.310

Haesler, E., Lyon, X., Pruvot, E., Kappenberger, L., and Hayoz, D. (2004). Confounding effects of heart rate on pulse wave velocity in paced patients with a low degree of atherosclerosis. J. Hypertens. 22, 1317-1322. doi: 10.1097/01.hjh.0000125447.28861.18 
Kim, Y. S., Seifert, T., Brassard, P., Rasmussen, P., Vaag, A., Nielsen, H. B., et al. (2015). Impaired cerebral blood flow and oxygenation during exercise in type 2 diabetic patients. Physiol. Rep. 3:e12430. doi: 10.14814/phy2.12430

Lantelme, P., Mestre, C., Lievre, M., Gressard, A., and Milon, H. (2002). Heart rate: an important confounder of pulse wave velocity assessment. Hypertension 39, 1083-1087. doi: 10.1161/01.HYP.0000019132.41066.95

Lewis, S. F., Taylor, W. F., Graham, R. M., Pettinger, W. A., Schutte, J. E., and Blomqvist, C. G. (1983). Cardiovascular responses to exercise as functions of absolute and relative work load. J. Appl. Physiol. Respir. Environ. Exerc. Physiol. 54, 1314-1323. doi: 10.1152/jappl.1983.54.5.1314

Mackenzie, I. S., McEniery, C. M., Dhakam, Z., Brown, M. J., Cockcroft, J. R., and Wilkinson, I. B. (2009). Comparison of the effects of antihypertensive agents on central blood pressure and arterial stiffness in isolated systolic hypertension. Hypertension 54, 409-413. doi: 10.1161/HYPERTENSIONAHA.109. 133801

Martina, J. R., Westerhof, B. E., van Goudoever, J., de Beaumont, E. M., Truijen, J., Kim, Y. S., et al. (2012). Noninvasive continuous arterial blood pressure monitoring with Nexfin(R). Anesthesiology 116, 1092-1103. doi: 10.1097/ALN.0b013e31824f94ed

McMichael, J. (1944). Clinical aspects of shock. J. Am. Med. Assoc. 124, 275-281. doi: 10.1001/jama.1944.02850050007003

Michard, F. (2005). Changes in arterial pressure during mechanical ventilation. Anesthesiology 103, 419-428. doi: 10.1097/00000542-200508000-00026

Pan, A. W., Chen, Y. L., Chung, L. I., Wang, J. D., Chen, T. J., and Hsiung, P. C. (2012). A longitudinal study of the predictors of quality of life in patients with major depressive disorder utilizing a linear mixed effect model. Psychiatry Res. 198, 412-419. doi: 10.1016/j.psychres.2012.02.001

Pinheiro, J., B. D., DebRoy, S., and Sarkar, D., and, R Core Team (2014). nlme: Linear and Nonlinear Mixed Effects Models. R package version 3.1-117.

R Core Team (2014). R: A Language and Environment for Statistical Computing. R Foundation for Statistical Computing.

Rimoldi, S. F., Messerli, F. H., Cerny, D., Gloekler, S., Traupe, T., Laurent, S., et al. (2016). Selective heart rate reduction with ivabradine increases central blood pressure in stable coronary artery disease. Hypertension 67, 1205-1210. doi: 10.1161/HYPERTENSIONAHA.116.07250

Ryan, K. L., Rickards, C. A., Hinojosa-Laborde, C., Cooke, W. H., and Convertino, V. A. (2012). Sympathetic responses to central hypovolemia: new insights from microneurographic recordings. Front. Physiol. 3:110. doi: 10.3389/fphys.2012.00110
Secher, N. H., and Van Lieshout, J. J. (2016). "Hypovolemic shock," in Clinical Fluid Therapy in the Perioperative Setting, 2nd Edn., ed R. G. Hahn (Cambridge: Cambridge University Press), 222-231.

Selvaraj, S., Steg, P. G., Elbez, Y., Sorbets, E., Feldman, L. J., Eagle, K. A., et al. (2016). Pulse pressure and risk for cardiovascular events in patients with atherothrombosis: from the REACH registry. J. Am. Coll. Cardiol. 67, 392-403. doi: 10.1016/j.jacc.2015.10.084

Torng, P. L., Chang, W. C., Hwang, J. S., Hsu, W. C., Wang, J. D., Huang, S. C., et al. (2007). Health-related quality of life after laparoscopically assisted vaginal hysterectomy: is uterine weight a major factor? Qual. Life Res. 16, 227-237. doi: 10.1007/s11136-006-9123-y

Verbeke, G., and Molenberghs, G. (2000). Linear Mixed Models for Longitudinal Data. New York, NY: Springer-Verlag.

Westerhof, B. E., van den Wijngaard, J. P., Murgo, J. P., and Westerhof, N. (2008). Location of a reflection site is elusive: consequences for the calculation of aortic pulse wave velocity. Hypertension 52, 478-483. doi: 10.1161/HYPERTENSIONAHA.108.116525

Westerhof, N., and Westerhof, B. E. (2013). Forward and backward pressure waves in the arterial system do represent reality. J. Physiol. 591, 1167-1169. doi: 10.1113/jphysiol.2012.249763

Wilkinson, I. B., MacCallum, H., Flint, L., Cockcroft, J. R., Newby, D. E., and Webb, D. J. (2000). The influence of heart rate on augmentation index and central arterial pressure in humans. J. Physiol. 525, 263-270. doi: 10.1111/j.1469-7793.2000.t01-1-00263.x

Conflict of Interest Statement: : BW previously worked for Edwards Lifesciences Corp. They, however, had no say in any of the data or work presented.

The other authors declare that the research was conducted in the absence of any commercial or financial relationships that could be construed as a potential conflict of interest.

Copyright (c) 2018 van Der Ster, Sperna Weiland, Westerhof, Stok and van Lieshout. This is an open-access article distributed under the terms of the Creative Commons Attribution License (CC BY). The use, distribution or reproduction in other forums is permitted, provided the original author(s) and the copyright owner are credited and that the original publication in this journal is cited, in accordance with accepted academic practice. No use, distribution or reproduction is permitted which does not comply with these terms. 